Results Total sample size was 2,331 and 2,282 in Rounds 1 and 2, respectively. In Round 1, syphilis (6.1\%), HIV (9.5\%) and HSV-2 $(31.3 \%)$ was highest among clients soliciting FSWs from brothels. CT/GC (6.6\%) was highest among clients of FSWs based in lodges. In Round 2, syphilis (3.2\%), HIV (6.9\%) and HSV-2 (20.5\%) were highest amongst clients soliciting in lodges, brothels and public places, respectively. Statistical significance was reached for HIV $(p=0.003)$ and CT/GC $(p=0.011)$ by site in Round 1 only. Differences by solicitation site in Round 1 remained significant when adjusted for district. Between rounds, prevalence decreased in all solicitation sites, with the exception of syphilis $(2.0 \%$ to $3.2 \%)$ and HIV $(0.6 \%$ to $1.1 \%)$ among clients frequenting lodges.

Conclusions Clients frequenting brothels had the highest HIV prevalence. Further study of the increasing prevalence of syphilis and HIV among clients frequenting lodges is warranted. Approaches focussing on where clients solicit FSWs, rather than identifying clients per se can help inform programming activities.

\section{P6.005 MULTIDISCIPLINARY APPROACH TO MANAGING A SYPHILIS OUTBREAK IN SOUTHEND-ON-SEA, ESSEX UK}

doi:10.1136/sextrans-2013-051184.1159

'K A Fernando, ${ }^{2} \mathrm{~S}$ Matthews, ${ }^{1} \mathrm{H}$ Jaleel, ${ }^{3} \mathrm{~S}$ Salimee. 'Department of Sexual Health \& HIV Medicine, Southend University Hospital NHS Foundation Trust, Essex, UK; ${ }^{2}$ Department of Public Health, Essex Primary Care Trust, Essex, UK; ${ }^{3}$ Essex Health Protection Unit, Health Protection Agency, Essex, UK

Background In 2012 the Essex Health Protection Unit of the Health Protection Agency(HPA) was alerted to a significant rise in syphilis in Southend. 27 cases were diagnosed in 2011 and 15 in the first half of 2012, compared to just 10 in 2010, indicating a greater than $50 \%$ rise. All were in men who have sex with men. In England as a whole, in 2011, 2915 syphilis cases were diagnosed, reflecting a less modest $10 \%$ rise compared to 2010 .

Importantly, Southend is a high prevalence UK area of HIV, with a population prevalence of 2.76 per 1000 . 6 of 27 individuals diagnosed with syphilis in Southend in 2011 were HIV-infected.

Aim A multidisciplinary incident management team (MDT) was established to investigate and establish measures to control the outbreak. The MDT includes members of the local health authority, public health department, HPA and genitourinary medicine(GUM) department. The MDT agreed to monitor GUM and diagnostic laboratory activity to inform testing and prevention strategies.

Interventions Local media press releases alerting the public of the syphilis outbreak were produced. Such information advised on sexual health promotion, irrespective of age or perceived risk. The Gay Essex Men's Support Group, Terrence Higgins Trust, and local contraception services were also alerted and encouraged to use existing networks to raise awareness.

Annual use of one of 6 pharmacy campaigns to raise STI awareness was implemented. Dissemination (verbally and writtenintranet) of information on STI trends and management was well received by local general practitioners.

Conclusions Firstly, local accurate reporting confirmed the increase in syphilis diagnoses, and enabled timely review and public health action. Secondly, good interagency and multidisciplinary working resulted in productive collaborative responses. And finally, the use of existing sexual health and MSM social/support networks enabled access to 'harder to reach' populations with regards to STI health promotion.

\section{P6.006 COUNTING THE PENNIES: RATIONAL PRESCRIBING WITHIN THE CONTEXT OF THE NATIONAL CHLAMYDIA SCREENING PROGRAMME}

doi:10.1136/sextrans-2013-051184.1160

'K M Turner, ${ }^{1} \mathrm{P}$ Horner, ${ }^{1} \mathrm{I}$ Vincent, ${ }^{2} \mathrm{E}$ Adams, ${ }^{1} \mathrm{~J}$ Macleod. ' University of Bristol, Bristol, UK: ${ }^{2}$ Aquarius Population Health, London, UK
Background In 2010/11 the National Chlamydia Screening Programme diagnosed over 90,000 cases of chlamydia in England. The current BASHH guidelines recommend either Azithromycin or doxycycline. Concerns have been raised questioning the efficacy of Azithromycin in routine practise.

Aims The aim of our study was to analyse whether current prescribing is in line with the criteria for rational prescribing and to estimate the cost and efficacy implications of a change in prescribing practise.

Methods We searched for published data on clinical effectiveness (both in randomised controlled trials and in practise), cost, compliance, universal use, acceptability, antimicrobial resistance and safety profiles.

We developed a simple costing tool to estimate the potential cost implications of prescribing practise and clinical effectiveness on the total treatment cost and the cost per infection treated.

Findings Until 2012, Azithromycin cost £9.65 per 4-tab 250mg pack in comparison with $£ 1.70$ per 28 capsule $50 \mathrm{mg}$ pack (BNF 2011-12). The cost of Azithromycin is now £1.50. The most recent findings in practise have suggested that the clinical effectiveness of Doxycycline may be superior to Azithromycin.

Interpretation

In health economic terms, if Doxycycline is clinically superior and costs less than Azithromycin, then Doxycycline regimens dominate. This could have saved significant costs in 2011/12 (up to $£ 500,000$ ). Now the costs are broadly equivalent, providing that the cheaper form of Azithromycin is prescribed. Doxycycline is also a valid choice of therapy, and extended regimens of Azithromycin could now also be considered without being prohibitively expensive.

Policy recommendation

Within the context of treatment of asymptomatic, uncomplicated chlamydia diagnosed through screening, Azithromycin is likely to continue to be the drug of choice. The process for implementing changes to prescription should be made more streamlined and transparent, so that evidence can be more rapidly translated into cost-savings.

\section{P6.007 ADDRESSING ENDEMIC RATES OF STI IN REMOTE ABORIGINAL COMMUNITIES IN AUSTRALIA USING QUALITY IMPROVEMENT AS A KEY STRATEGY: THE STRIVE STUDY}

doi:10.1136/sextrans-2013-051184.1161

1,2J Ward, ${ }^{2} \mathrm{R}$ Guy, ${ }^{2} \mathrm{~L}$ Garton, ${ }^{3} \mathrm{~B}$ Silver, ${ }^{3} \mathrm{D}$ Taylor-Thomson, $4,2 \mathrm{~B}$ Hengel, ${ }^{2} \mathrm{~J}$ Knox, ${ }^{2} \mathrm{~S}$ McGregor, ${ }^{3,5} \mathrm{~A}$ Rumbold, ${ }^{2} \mathrm{~J}$ Kaldor. ${ }^{\prime} B a k e r$ IDI, Alice Springs, Northern Territory, Australia; ${ }^{2}$ The Kirby Institute, University of New South UK, Sydney, Australia; 'Menzies School of Health Research, Darwin, Northern Territory, Australia; ${ }^{4}$ Apunipima Cape York Health Council, Cairns, Queensland, Australia; ${ }^{5}$ University of Adelaide, Adelaide, South Australia, Australia

Background Remote Aboriginal communities in Australia have substantially higher rates of chlamydia (CT), gonorrhoea (NG) and trichomoniasis (TV) diagnosis compared with non-Indigenous people, despite many years of programme and policy aimed at reducing disparity.

Methods 'STRIVE' is a stepped wedge cluster randomised controlled trial, underway in 68 remote Aboriginal communities. The primary objective is to assess if a sexual health quality improvement programme within primary care clinics improves STI management and in turn reduces STI prevalence. Data is extracted from both patient management systems and from laboratories. To evaluate the impact of the trial, the largest STI prevalence study in Aboriginal people has been undertaken twice.

Results In the first year of the trial, testing rates have improved overall by $27 \%$ at 21 sites and by $50 \%$ or more at 11 sites. In 2011 , baseline prevalences for CT and NG $(n=2483)$ and TV $(n=1848)$ among 16-34 year olds were $9 \%, 7 \%$ and $13 \%$ respectively. Highest 
prevalences were reported among 16-19 year olds for CT 13\% (95\% CI; 10.8-16.4), NG, 12\% (95\% CI; 9.7-15.1) and TV, 17\%(95\% CI; 13.7-21.1). There were 17,848 STI tests conducted in 2010 and among females aged 16-34; 33.3\% had $\geq 1$ STI (highest in 16-19 year olds: $48.9 \%$ ) and $21.3 \%$ of males had $\geq 1$ STI (highest in 16-19 year olds:33.4\%). The most frequent co-infection was CT and NG which was found in $3.4 \%$ of females (highest in 16-19 year olds: $8.6 \%$ ) and $3.9 \%$ of males (highest in 16-19 year olds:10.1\%).

Discussion STRIVE has provided information not previously available in regard to a comprehensive epidemiological picture of STI morbidity and health service responses in remote Aboriginal communities and highlights work required especially among young people. The results of STRIVE may be of relevance to other areas globally with STI endemic rates.

\section{P6.008 EXTERNAL QUALITY ASSURANCE WITH DRIED TUBE SPECIMENS (DTS) FOR POINT OF CARE SYPHILIS AND HIV TESTS: EXPERIENCE IN AN INDIGENOUS POPULATIONS SCREENING PROGRAMME IN THE BRAZILIAN AMAZON}

doi:10.1136/sextrans-2013-051184.1162

'A S Benzaken, ${ }^{2} \mathrm{M}$ Bazzo, 'E Galban, ${ }^{1}$ P Pinto, ${ }^{2} \mathrm{C}$ L Nogueira, ${ }^{2} \mathrm{~L}$ Golfetto, ${ }^{3} \mathrm{~N}$ S Benzaken, ${ }^{4} \mathrm{~K}$ Sollis, ${ }^{4} \mathrm{D}$ Mabey, ${ }^{4} \mathrm{R}$ Peeling. ${ }^{1}$ Alfredo da Matta Foundation, Manaus, Brazil; ${ }^{2}$ Federal University of Santa Catarina, Florianópolis, Brazil; ${ }^{3}$ Nilton Lins University, Manaus, Brazil; ${ }^{4}$ London School of Hygiene and Tropical Medicine, London, UK

Background The availability of point of care(POC) tests for infectious diseases has revolutionised the provision of health care for remote rural populations without access to laboratories. However, little attention has been given to quality assurance for POC tests. In a screening project that tested 45,226 adults of both sexes by 268 Health Care Workers(HCWs), in remote indigenous populations in the Amazon region of Brazil, where the overall prevalence of syphilis was $1.6 \%$, and of HIV 0.1\%, we evaluated the use of Dry Tube Specimens(DTS) for External Quality Assurance(EOA) for POC HIV and Syphilis tests. Methods The EOA programme was implemented from March 2010 to March 2011 using DTS panels developed by a reference laboratory, containing samples with negative and positive results at different antibody concentrations, for HIV and Syphilis infection. These were re-suspended and tested in the communities by each HCW. We also conducted stability tests for the panels at the reference laboratory.

Results Results from $268 \mathrm{HCWs}$, responsible for implementing the POC tests at six Indigenous District(DSEI) participated in the EOA programme, showed a concordance rate of $90 \%$ for syphilis and $93 \%$ for HIV (Kappa coefficients of 0.74 and 0.78 respectively) with reference laboratories for a total of 1,608 determinations. The highest rate of inaccurate diagnoses occurred in positive samples of very low antibody concentration (40\% for syphilis and $11.9 \%$ for HIV). The stability tests showed that titers were stable for up to one week at $30^{\circ} \mathrm{C}$ in dry conditions.

Conclusion The results show that errors in the interpretation of POC test results were identified by the EOA programme using DTS. The use of POC tests for syphilis and HIV is now recommended as a policy by the Brazilian government. EOA/using DTS can help to improve the quality of these screening programmes and is already being implemented nationally.

\section{P6.009 IMPROVED TIMELY DIAGNOSIS OF HIV RELATED TO THE POLICY OF EXPANDING ACCESS TO DIAGNOSIS IN BRAZIL}

doi:10.1136/sextrans-2013-051184.1163

H Bernal, A A Pereira, A Pires, A R Costa, E Z Ayer, A Bolzan, M R Arruda, A I Sousa, A P Pascom, M A Freitas. Ministry of health, Brasilia, Brazil

Improved timely diagnosis of HIV related to the policy of expanding access to diagnosis in Brazil.
Background In the last years the Brazilian Ministry of Health expanded the use of rapid tests (RT) for HIV. RT is especially useful for pregnant women, vulnerable populations, people with limited access to health services and at national testing campaigns. This policy aims to ensure timely diagnosis and treatment, what will impact directly on morbimortality of people living with HIV/aids (PLWHA).

Methods National network consists of 90 national public laboratories responsible for performing CD4 count for the public health system. Between 2005 and 2012, 3.7 million tests were registered in its database. Based on the first CD4 count registered, diagnosis was classified as very late $\left(<200\right.$ cells $\left./ \mathrm{mm}^{3}\right)$, late (between 200 and 350 cells $/ \mathrm{mm}^{3}$ ) and timely $\left(>350\right.$ cells $\left./ \mathrm{mm}^{3}\right)$.

Results Our database was comprised of 321 thousand PLWHA > 18 y.o. who started on follow up in the public health system between 2005 and 2012. In 2005, 30.6\%, 21.6\% and 47.7\% of PLWHA were diagnosed very late, late and timely, respectively. In 2012 there was a significant decrease in very late and late diagnosis and an increase in timely diagnosis: 29\%, 18\% and 53\% respectively. Conclusion The policy of expanded access to rapid test certainly contributed to these results. Indeed, there was an increase in rapid tests supply: from 509,180 in 2005 to 3,750,000 in 2012, facilitating that PLWHA are diagnosed with higher CD4 counts, what is confirmed by the increase of median first CD 4 count from 335 in 2005 to 365 cells $/ \mathrm{mm}^{3}$ in 2012 .

\section{P6.010 IMPLEMENTATION OF VIA FOR CERVICAL CANCER SCREENING IN A SEXUALLY TRANSMITTED INFECTION CLINIC IN LILONGWE, MALAWI}

doi:10.1136/sextrans-2013-051184.1164

1,2M Hosseinipour, 'B Ndalama, 1,2N E Rosenberg, 'G Kamanga, ${ }^{1} \mathrm{C}$ Mapanje, ${ }^{3} \mathrm{~S}$ Phiri, ${ }^{2}$ W C Miller, ${ }^{1}, 2 \mathrm{~F}$ Martinson, ${ }^{1,2} \mathrm{I}$ Hoffman. ${ }^{1}$ UNC Project, Lilongwe, Malawi; ${ }^{2}$ University of North Carolina, Chapel Hill, NC, United States; ${ }^{3}$ Lighthouse Trust, Lilongwe, Malawi

Background Cervical cancer is the most common female cancer in Malawi with high mortality. Cervical cancer can be averted if precancerous lesions are detected early and treated. Visual Inspection with Acetic Acid (VIA) is an effective screening method for resourcelimited settings. In an STI Clinic in Lilongwe, Malawi, VIA screening implementation was assessed through the NIH-funded Medical Education Partnership Initiative. In this setting, 20\% of women have reactive VIA results.

Methods Females attending the Kamuzu Central Hospital STI Clinic from October 2012 to January 2013 were included. Screening was recommended for women $25-45$ years and women $<25$ years at clinician discretion. We explored the proportion of women who were screened, characterised write-in reasons for non-screening, and conducted binomial regression to explore screening predictors.

Results During this 3.5 month period, 956 women presented for 1240 STI clinic visits. Four percent of women $<25$ and $19 \%$ of women 25-45 received VIA screening. Among women 25-45, common reasons for not screening included postponement (19\%) (often due to STI treatment or cervical pain), recent screening (14\%), menses $(8 \%)$, and pregnancy (3\%). Few refused (3\%). Many did not have reasons recorded (44\%). Screening was less common among women presenting through partner-referral $(0.3$; CI $0.1,1.1)$ and among women who did not receive pelvic exams as part of STI assessment (0.6, CI: 0.3, 0.9).

Conclusions In this high risk setting, VIA implementation was feasible and acceptable. On the day of presentation, many women were not screened due to cervical pain, STI treatment, menses, or pregnancy. Ensuring that these women return for screening is important. Offering VIA to all women is essential, even those not otherwise receiving a pelvic exam. Clear guidelines on whether and 